\title{
Butyrophilin-like 9 regulates immune infiltration and serves as a prognostic marker in lung adenocarcinoma
}

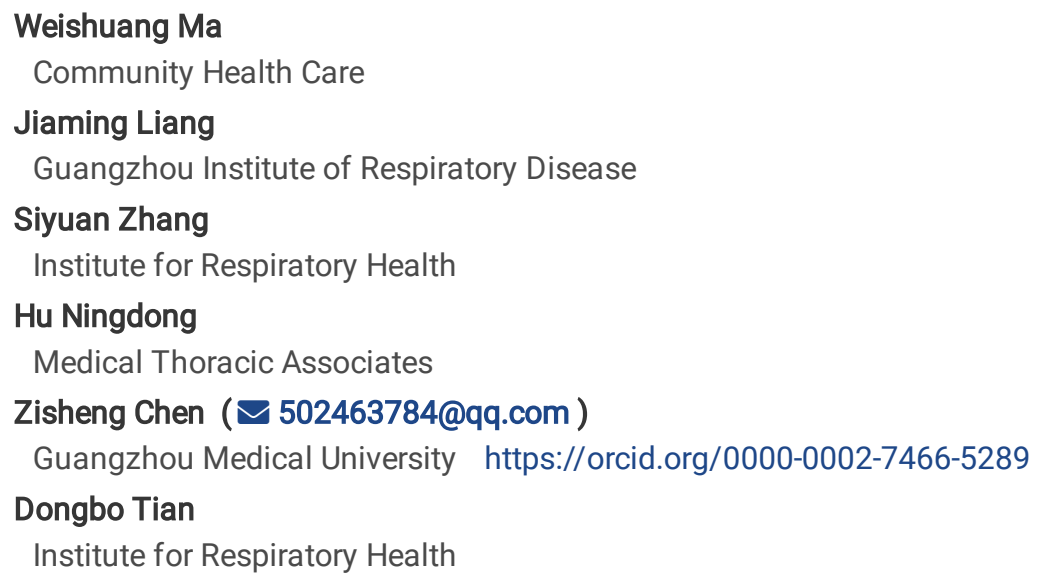

\section{Primary research}

Keywords: Butyrophilin-like 9, B cells, Dendritic cells, Prognosis, Lung adenocarcinoma

Posted Date: October 21st, 2020

DOI: https://doi.org/10.21203/rs.3.rs-92631/v1

License: (c) (i) This work is licensed under a Creative Commons Attribution 4.0 International License. Read Full License 


\section{Abstract}

Background: Butyrophilin (BTN) and butyrophilin-like (BTLN) belong to immunoglobulin superfamily, and also pertain the B7 co-stimulatory molecules family, which has multiple roles in immune modulation. Whether the BTNL9 expression in lung adenocarcinoma (LUAD) correlates with outcome has not been evaluated.

Materials and methods: Oncomine and GEPIA were used to analyze the BTNL9, while its mRNA expression in LUAD and corresponding adjacent tissues was investigated using TIMER. The clinical prognosis of BTNL9 was assessed in the GEPIA, Kaplan-Meier plotter, and OncoLnc. Besides, the correlation between BTNL9 and tumor-infiltrating immune cells (TILs) was analyzed using TIMER and GEPIA. The correlation between BTNL9 expression and drug response was analyzed using CARE.

Results: BNL9 expression was significantly low in LUAD. Low BTNL9 expression was associated with poor OS, and its expression was found to be regulated by both epigenetic regulation and post-transcriptional modification. BTNL9 expression was significant positively correlated with ImmuneScore and ESTIMATEScore. Moreover, BTNL9 expression was positively correlated with infiltrating levels of B cells, $\mathrm{CD} 4+\mathrm{T}$, and macrophages, but Kaplan-Meier analysis showed that BTNL9 expression in B cells and DCs was significantly associated with OS. Furthermore, BTNL9 expression has significant positive CARE scores.

Conclusions: Low BTNL9 expression can prevent the infiltration of naïve B cells and DCs in the tumor microenvironment and worsen the outcome in LUAD patients. Besides, these findings also suggest the potential role of BTNL9 as a prognostic biomarker and a new immunotarget.

\section{Background}

GLOBOCAN 2018 estimated the number of new lung cancer cases and mortality in lung cancer to be 2,093,876 and 1,761,007, respectively[1]. Besides, lung cancer accounts for $11.6 \%$ of the total cancer cases and $18.4 \%$ of the total cancer deaths, hence it is considered the most frequently diagnosed cancer[1]. In China, lung cancer is also the most commonly diagnosed and a leading cause of cancer-related deaths [2]. LUAD and lung squamous cell carcinoma (LUSC) are the most common histological subtypes of non-small cell lung cancer (NSCLC), accounting for approximately $85 \%$ of all the subtypes[3]. LUAD has been considered the most prevalent histopathological subtype since 1985[4]. The development of immunotherapy has significantly improved the prognosis of LUAD patients. However, the 5-year survival rate of LUAD remains disappointing[4]. Therefore, new immuno-targets and treatment strategies require to be urgently developed.

Butyrophilin (BTN) and butyrophilin-like (BTLN) belong to the immunoglobulin superfamily and are also related to the B7 family of costimulatory molecules, which play a significant role in the T cell suppression, regulation of epithelial cell and T cell interplays[5]. The human BTN genes are located in the MHC class $\otimes$ domain of the short arm of chromosome 6 (6p22.1) and include three subfamilies, which form phylogenetically related groups: BTN1, BTN2, and BTN3. BTN1A1 belongs to BTN1, BTN2A1, BTN2A2, and BTN2A3 (BTN2A3P) belong to BTN2, and BTN3A1, BTN3A2, and BTN3A3 belong to BTN3 subfamilies. Moreover, butyrophilin-like proteins (BTNL: BTNL2, BTNL3, BTNL8, BTNL9, and BTNL10) and SKINT-like (SKINTL) are classified in the family of butyrophilins[5-7].

The functions of BTN and BTNL family members have not yet been elucidated, especially in tumor microenvironment (TME). BTN1A1, BTN2A2, and BTNL2 proteins through cell cycle arrest have been reported to show an inhibitory effect on CD $4+T$ and CD8 + T cell proliferation[6]. BTN3 family exerts an immunosuppressive effect, which inhibits T cell activation. However, the functions of BTNL3, BTNL8, and BTNL9 remain unknown[5].

In this study, we investigated the relationship between the expression of BTN and BTNL and prognosis in LUAD patients[8]. Gene Expression Omnibus[9], TIMER[10], KM plotter[11], UALCAN[12], OncoLnc[13], Oncomine[14], TissGDB[15]among other databases, has been used to screen for BTN or BTLN molecules, and to further explore their signal networks and tumor-immune interactions, providing a theoretical basis for precise prognosis and treatment of LUAD.

\section{Materials And Methods}

\section{BTNL9 mRNA expression in LUAD}

The "Tidyverse"[16] and "Table1" packages of R software[17] (version 4.0.1) were used to extract the clinical parameters of the LUAD dataset in TCGA, including age, gender, race, TNM staging, ECOG score, EGFR mutation, KRAS mutation, and radiotherapy information. 


\section{GEPIA database analysis}

Search the expression of BTN and BTNL family genes in LUAD in the GEPIA database (http://gepia2.cancer-pku.cn/\#index)[18]. The conditions were set as follows: $|\log 2 \mathrm{FC}|$ Cutoff $=1$, $p$-value Cutoff=0.01, $\log 2(T P M+1)$ for log-scale, Jitter Size $=0.4$ and Match TCGA normal and GTEx data. The overall Survival setting conditions were: Group Cutoff selects median, Cutoff-High=50\%, and Cutoff-Low=50\%. BTN or BTNL molecules that meet the significant difference between LUAD and adjacent tissue were only BTNL8 and BTNL9. Then survival analysis and select the molecule with significant differences, and the result is only BTNL9 in LUAD. BTNL9 was confirmed through the survival analysis of the three databases KM plotter (http://www.kmplot.com)[11], UALCAN (http://ualcan.path.uab.edu/index.html)[12] and OncoLnc (http://www.oncolnc.org/)[13], and subsequent network analysis including immune infiltrates were presented. GEPIA was used to analyze the correlation between BTNL9 and methyltransferase expression, B, and DC cell immune infiltration.

\section{Oncomine database analysis}

The mRNA expression of BTNL9 in different tumors was identified using the Oncomine database (https://www.oncomine.org/)[14]. The default setting parameters of the database were used, with the threshold parameters set as a P-value of 0.01 , a fold change of 1.5 , and a top $10 \%$ gene ranking.

\section{TissGDB database analysis}

TissGDB is the tissue-specific gene annotation database in cancer (https://bioinfo.uth.edu/TissGDB/index.html? csrt=17567836353851237153)[15]. Forest plot of Cox proportional hazard ratio (HR) and 95\% $\mathrm{Cl}$ of overall survival (OS) and relapse-free survival (RFS) were performed in 28 cancer types .

\section{TIMER database analysis}

TIMER is a comprehensive resource for systematical analysis of immune infiltrates across diverse cancer types (http://timer.compgenomics.org/)[10, 19]. Using Gene_DE module, BTNL9 mRNA expression was calculatedin cross-carcinoma(*: P-value < 0.05; **: P-value $<0.01$; $* * *$ : P-value <0.001). The expression of BTNL9 in LUAD and its correlation with the six immune infiltration cells, namely B cells, CD4+ T cells, CD8+ T cells, macrophages, neutrophils, and dendritic cells, used as the Gene and Survival module. Gene_Corr module was used to analyze the correlation between BTNL9 expression and B and DC cells[20].

\section{StarBase database analysis}

We used three databases: miRMap (https://mirmap.ezlab.org/)[21], TargetScan (http://www.targetscan.org/vert_72/)[22], and miRWalk (http://mirwalk.umm.uni-heidelberg.de/)[23] to predict the miRNAs that could bind to BTNL9, consider the intersection, verify in the Starbase database (http://starbase.sysu.edu.cn/panCancer.php)[24], and select miRNAs with significant differences between co-expression and overall survival.

\section{Sangerbox database analysis}

Sangerbox (http://sangerbox.com/Index) was developed by Hangzhou Mugu Technology Co., Ltd. It mainly uses public data or existing data for immune-related analysis and directly issues a set of charts and analysis reports. Sangerbox uses the R software ESTIMATE package to analyze the immune score and stromal score of each TCGA tumor sample, and GSEA to analyze the enrichment for the KEGG and HALLMARK pathways in the BTNL9 high and low expression groups. Analysis of the relationship between BTNL9 and TMB was determined using Spearman correlation analysis.

\section{Statistical Analysis}

The boxplots of BTNL9 expression were extracted from GEPIA. Overall survival was determined from GEPIA, KM plotter, UALCAN, and OncoLnc databases. The expression of BTNL9 across cancer species was extracted in TIMER. RFS and OS HR were extracted from TissGDB database. The correlation between the miRNA and BTNL9 expression was determined in Starbase, as well as the expression of miRNA on the survival of LUAD. BTNL9 expression and single cancer cell biological behavior of LUAD was performed by Pearson correlation analysis. The correlation between BTNL9 expression, the 6 immune cells, and survival analysis was assessed in TIMER. In all the above analyses, $\mathrm{P}<0.05$ was consideredstatistically significant.

\section{Results}


The "Tidyverse" and "Table1" packages of R software (version 4.0.1) was used to extract the clinical parameters of the LUAD data set in TCGA. Further, we analyzed the age, gender, race, TNM staging, ECOG score, EGFR mutations, KRAS mutations, and radiotherapy information of the two groups of patients with high and low expression of BTNL9. There is no significant difference in two groups (data not shown), and we organized them into a table for descriptive analysis (Table 1).

\section{BTNL9 is the key gene of butyrophilins in LUAD}

The expression of BTNL8 and BTNL9 were significantly different in LUAD, and both tumor tissues were significantly lower than normal tissues (Figure 1A). BTNL9 significantly correlated with the clinical stage, N stage and P53 mutation, while BTNL8 significantly correlated with the clinical stage and N stage (Figure 1B). However, survival analysis revealed that BTNL8 was not significantly correlated with OS, while BTNL9 was significantly correlated with OS in LUAD (Figure 1C). PrognoScan[25] analysis of the NSCLC dataset found that BTNL9 expression was significantly correlated with RFS and OS in the GSE31210 study, and BTNL9 expression was significantly correlated with OS in GSE3141 study (Supplementary Table 1). KM plotter, UALCAN, and OncoLnc were used to validate the findings, and the results showed that high expression of BTNL9 significantly improved the OS of LUAD (Figure 1D). Therefore, we regarded BTNL9 as the key gene of BTN and BTNL family in LUAD.

\section{Expression of BTNL9 in pan-cancers and its prognostic correlation}

We analyzed the expression of BTNL9 in pan-cancer, and analysis of pan-cancers from the Oncomine database showed that BTNL9 expression was significantly reduced in breast cancer, one dataset of colon cancer, lung cancer, kidney cancer, and crabtree uterus cancer compared with the normal tissue. However, BTNL9 expression significantly increased in brain and CNS cancer, four datasets of colorectal cancer, esophageal cancer, leukemia, and lymphoma (Figure 2A and Supplementary Table 2). We further examined BTNL9 expression using TCGA RNA sequencing data (TIMER). As shown in Figure 2B, BTNL9 expression was significantly reducedin Bladder Urothelial Carcinoma (BLCA), Breast invasive carcinoma (BRCA), Cholangio carcinoma (CHOL), Esophageal carcinoma (ESCA), Glioblastoma multiforme (GBM), Head and Neck squamous cell carcinoma (HNSC), Kidney renal papillary cell carcinoma (KIRP2), LUAD, and LUSC, but significantly increasedin Colon adenocarcinoma (COAD), Kidney Chromophobe (KICH), and Kidney renal clear cell carcinoma (KIRC). The TissGDB database analysis revealed that the correlation of BTNL9 expression with LUAD's RFS HR was $0.87[95 \% \mathrm{Cl}(0.79,0.96)]$, and the OS HR was $0.87[95 \% \mathrm{Cl}(0.8,0.96)]$ (Figure 2C, 2D).

\section{Upstream and downstream regulatory network of BTNL9}

In previous study, we found that the expression of BTNL9 in LUAD was significantly lower compared with the normal tissues. DNA methylation is related to gene expression. Methyltransferases (DNMTs) including DNMT1, DNMT2, DNMT3A, and DNMT3B. Analysis of the GEPIA database revealed that the expression of BTNL9 in normal lung tissue was significantly positively correlated with DNMTs $(r=0.35, P=0.059)$, but there was no correlation in $\operatorname{LUAD}(r=-0.019, P=0.67)$ (Figure $3 A, 3 B)$, suggesting that DNA methylation may be involved in the pathogenesis of LUAD. We further analyzed the upstream regulation of BTNL9, predicted miRNAs that bind to BTNL9 through miRMap[21], TargetScan[26], and miRWalk[23], and considered the intersection of the 3 databases to obtain 248 miRNAs (Figure 3C). The miRNAs were verified in Starbase[24] database and found that, hsa-miR-30b-3p, hsa-miR- 4709-3p and hsa-miR-6514-39 were significantly positively correlated with BTNL9 expression ( $r=0.312, P=5.25 E-13, r=0.277, P=1.74 E-10$, and $r=0.103, P=0.02$, respectively), and the 3 miRNAs were highly expressed and significantly prolonged the OS of $L U A D(H R=0.66, P=0.0058, H R=0.63, P=0.0023$, and $H R=0.73$, $\mathrm{P}=0.036$, respectively) (Figure 3D). Furthermore, we predicted the IncRNA binding to BTNL9 through LncMap[27] database and obtained 18 LncRNAs (Supplementary Table 3), which were verified and predicted the OS correlation in these LncRNAs through LncACTdb2.0[28] database. LncRNA AP001462.6 was found to bind to BTNL9, and the high expression of AP001462.6 significantly prolonged the OS in LUAD ( $P=0.049)$ (Figure 3E).

Moreover, we analyzed the protein network that binds to BTNL9 through the STRING[29] database and found that there were 7 binding proteins (Figure 3F). The top 2 binding proteins were predicted using the cytoHubba module of Cytoscape[30] (Figure 3G). The proteins that bind to BTNL9 were predicted to be HTRA4 and TM4SF19. HTRA4 gene encodes a member of the HtrA family of proteases, which may function as a secreted oligomeric chaperone protease to degrade misfolded secretory proteins[18]. We hypothesized that the low expression of BTNL9 in LUAD may be related to ubiquitination degradation. Analysis ofthe Ubibrowser[31] database revealed that E3 (MARCH8) ligases can bind the substrate BTNL9 (Confidence level is high, and score=0.805), and there is one potential E3 recognizing domain and two potential E3 recognizing motif (Figure 3H, 3l).

\section{Low BTNL9 expression significantly enriches proteasome and increases cancer malignancy}


To determine the biological function of BTNL9 expression on LUAD, the Sangerbox tool was used to divide the TCGA samples into high and low BTNL9 expression groups. Gene set enrichment analysis (GSEA) for KEGG and HALLMARK pathways were performed. The results revealed that the top 3 KEGGs pathways with high expression of BTNL9 were significantly enriched in vascular_smooth_muscle_contraction, phosphatidylinositol_signaling_system, and abc_transporters (Figure 4A). The top 4 KEGGs pathways associated with low expression of BTNL9 were parkinsons_disease, oxidative_phosphorylation, DNA replication, and proteasome (Figure 4B). GSEA for the HALLMARK pathway revealed that the top 3 pathways associated with high BTNL9 expression were bile_acid_metabolism, heme_metabolism, and wnt_beta_catenin_signaling, while the top 4 pathways associated with low BTNL9 expression were E2F_targets, glycolysis, myc_targets v1, and mTORC1_signaling (Figure 4C, 4D).

Metabolic reprogramming is a hallmark of cancer, and intrinsic and extrinsic factors contribute to metabolic phenotypes in tumors. As cancer develops from pre-tumor lesions to local, clinically obvious malignant tumors to metastatic cancer, the phenotype and dependence of metabolism also develop[32]. Through single-cell RNA (scRNA) analysis of LUAD in CancerSEA[33] database (Figure 4E), BTNL9 expression was found to be significantly negatively correlated with tumor malignant features including invasion $(r=-0.53, P<0.0001)$, metastasis $(r=-0.35, P=0.011)$, EMT $(r=-0.47, P=0.0006)$, proliferation $(r=-0.37, P=0.0086)$, Hypoxia $(r=-0.36, P=0.011)$, and DNA damage $(r=-0.34, P=0.017)$ (Figure 4F). Therefore,the low expression of BTNL9 promotes the malignant features of LUAD.

\section{Correlation between BTNL9 and markers of infiltrating immune cells and its prognosis}

Tumor mutation burden (TMB) is measured by the number of somatic mutations that occur at an average of $1 \mathrm{Mb}$ in the coding region (exon region) of the tumor cell genome (non-synonymous mutations). The total number of synonymous mutations indicates that the mutation types mainly include single nucleotide mutations (SNV) and small fragments of insertion/deletion (Indel) and other forms of mutations. Spearman's correlation test analyzed the correlation between BTNL9 and TMB in TCGA-LUAD and found that BTNL9 was significantly negatively correlated with TMB $(P=1.4 \mathrm{E}-9)$ (Figure 5A). Further, analysis of the somatic mutation pattern of BTNL9 in LUAD (Frame_Shift_Del, Missense, and Splice), revealed that the mutation frequency of BTNL9 in LUAD was 1.14\% (Figure 5B). Genetic mutations shape TME[34]. SangerBox tool used the R software package ESTIMATE to analyze the correlation between BTNL9 and stromal score. The results showed that BTNL9 was significantly positively correlated with ImmuneScore $(r=0.129, P=0.003)$ and ESTIMATEScore $(r=0.106$, $\mathrm{P}=0.016$ ). However, StromalScore was not significantly different (Figure $5 C, 5 D, 5 E$ ).

The correlation between BTNL9 and TME infiltrating immune cells was analyzed using TIMER[10] database and found that BTNL9 was negatively correlated with tumor purity $(r=-0.124, P=5.6 \mathrm{E}-03)$, and significantly positively correlated with $B$ cells $(r=0.24, P=8.88 E-8), C D 4+T$ $(r=0.283, P=2.24 E-10)$ and macrophages $(r=0.209, P=3.35 E-6)$ (Figure 5F). Moreover, survival analysis showed that high expression of BTNL9 in $B$ cells $(P=0)$ and $D C$ cells $(P=0.048)$ significantly prolonged the OS of $L U A D$ (Figure $5 G)$.

DC also have a trend of difference, and high DC expression of BTNL9 significantly prolonged OS in LUAD (Figure 5F, 5G). We conducted a detailed analysis of TME infiltrated DC and B cells using the TIMER database, which revealed that DC and its subtypes CDCs1 and CDCs2[35] were all associated with BTNL9 expression before and after purity adjustment. GEPIA database analysis found that normal lung tissue was not correlated with DC and its subtypes CDCs1 and CDCs2, but was significantly positively correlated with LUAD (Table 2), indicating that all DCs regulated by BTNL9 may participate in the LUAD immune response. $B$ cells are heterogeneous, and include two subtypes: naïve $B$ cells and plasma B cells[36]. TIMER analysis found that total B cells and naïve B cells were significantly related to BTNL9 expression before and after purity adjustment, but plasma B cells were not correlated to BTNL9 expression before and after purity adjustment. GEPIA analysis found that total B cells and naïve B cells were not correlated to BTNL9 expression in normal lung tissues, but significantly positively correlated in LUAD. Plasma B cells showed no correlation with BTNL9 in both normal tissues and LUAD (Table 2), indicating that BTNL9 may promote naïve B cell anti-tumor immune response.

\section{BTNL9 expression is to be associated with drug response}

Computational Analysis of Resistance (CARE) is a software that uses compound screening data to identify genome-scale biomarkers of targeted therapeutic response. According to the Pearson correlation between the gene expression profile of the cancer sample and the CARE scoring vector, the patient is predicted as a responder or a non-responder[37]. BTNL9 expression has significant positive CARE scores for many compounds in Cancer Cell Line Encyclopedia (CCLE), Genomics of Drug Sensitivity in Cancer (GDSC, previously named CGP), and The Cancer Therapeutics Response Portal (CTRP) cohorts, suggesting that loss of BTNL9 expression may promote drug resistance toward many targeted therapies (Figure 6, and Table 3).

\section{Discussion}


This study systematically analyzed the correlation between human BTNL9 and LUAD and normal adjacent tissues and revealed that BTNL9 can be considered the key gene of butyrophilins in LUAD. Through multiple database survival analysis, this study demonstrated that high BTNL9 expression significantly prolonged OS of LUAD. BTNL9 is significantly related to B cells, CD $4+T$, and macrophages in the TME of LUAD, while high expression of BTNL9 in B and DC cells significantly prolongs the OS of LUAD. GEPIA correlation analysis shows that BTNL9 is related to DC cells and naïve B cells but not plasma B cells in LUAD. Moreover, BTNL9 expression is associated with many targeted therapy response.

The low expression of BTNL9 in LUAD is regulated by unknown mechanisms. DNA methylation is the most common form of DNA modification, which plays an important role in normal cell physiology, increased DNA methylation, and loss of demethylation, which frequent in cancer. DNMTs play an important role during abnormal DNA methylation. Gene body hypermethylation may activate oncogenes, and promoter hypermethylation often causes the suppression of tumors[38].GEPIA analysis revealed that BTNL9 and DNMTs were significantly related to normal lung tissues, but not to LUAD (Fig. 3A, 3B). However, the molecular mechanism of DNMTs regulating BTNL9 in LUAD is unclear. miRNA and IncRNA are common non-coding RNAs, involved in tumor-promoting and suppressing, depending on the type of tumor[38]. We predicted that the 3 miRNAs (hsa-miR-30b-3p, hsa-miR-4709-3p, and hsa-miR-6514-39) were significantly positively correlated with BTNL9 in LUAD, and their high expression significantly prolongs OS (Fig. 3C, 3D), by exerting anti-cancer activity in LUAD. Hsu Y-L et al. reported that BTNL9 may act as a tumor suppressor in LUAD and may be regulated by hsa-miR-183-5p, but the specific regulatory network was not explained[39]. We further predicted that IncRNA AP001462.6 could bind to BTNL9, and the high expression significantly prolonged the OS of LUAD (Fig. 3E). Moreover, we analyzed the protein interaction network of BTNL9 and found that the interacting proteins played a role in immune regulation, protease hydrolysis, serine/threonine kinase regulation, etc. (Fig. 3F, 3G), and protease hydrolysis is related to ubiquitination degradation. The analysis also revealed that BTNL9 has a potential E3 recognizing domainbinding site (Fig. 3H, 3I). These indicates that BTNL9 in LUAD may be regulated by DNA methylation and non-coding RNA, and the protein may also be regulated by ubiquitination degradation after translation.

To understand the biological function of BTNL9 in LUAD, GSEA analysis was performed and revealed that low expression of BTNL9 was enriched in energy metabolism (oxidative_phosphorylation, glycolysis, myc_targets v1[40], and mTORC1_signaling[41]), DNA replication, and protease hydrolysis (Fig. 4A-4D). Metabolic reprogramming triggers selective gene amplification and a large gene family which drives cellular functions to promote cancer cell growth and proliferation[40]. The above functions were subsequently verified from a single cell perspective and found that BTNL9 expression was significantly negatively correlated with cancer cell malignant behaviors such as proliferation, invasion, EMT, metastasis, and hypoxia, etc. in LUAD (Fig. 4E, 4F). This indicates that BTNL9 may play an important and extensive tumor suppressor effect in LUAD.

We further analyzed the relationship between BTNL9 and TME and found that the mutation frequency of BTNL9 in LUAD was about 1.14\%, and BTNL9 was significantly negatively correlated with TMB (Fig. 5A, 5B). BTNL9 was significantly positively correlated with ImmuneScore and ESTIMATEScore (Fig. 5C, 5D, 5E). Previous studies have shown that high ImmuneScore and ESTIMATEScore significantly improve the prognosis of LUAD[42]. This shows that BTNL9 plays an important role in TME immune regulation. Moreover, we analyzed the correlation between BTNL9 and TILs and found that BTNL9 was significantly negatively correlated with tumor purity, while previous studies have found that low tumor purity is associated with poor prognosis[43]. Although BTNL9 is significantly related to B, CD4 + T, and macrophages, survival analysis showed that BTNL9 was only significantly related to B and DC cells (Fig. 5F, 5G).

DCs play a pivotal role in shaping the innateand adaptive immune response because they have the unique ability to initiate $T$ cell responses and differentiate them into effector lineages[35], while B cells have antigen presentation, cytotoxicity, and antibody production functions, which are an important part of adaptive immunity[44]. TIMER combined with GEPIA database analysis showed that the expression of BTNL9 on DCs (CDC1s and CDC2s) was not correlated with normal adjacent tissues, but was significantly correlated with LUAD tissues (Table 2). cDC1s can migrate to tumor-draining lymph nodes, activate and attract T cells, secrete cytokines, and present antigens in TME, thereby enhancing the function of local cytotoxic $T$ cells[45]. $c D C 2 s$ present antigens to $M H C \otimes$, activate $C D 4+T$ cells, and effectively polarize TILs into anti-tumor T helper cell 1 (Th1) or Th17 phenotype[46]. BTNL9 on B cells (naïve B cells) was not correlated with normal adjacent tissues but was significantly correlated with LUAD tissues, except for plasma B cells, suggesting that BTNL9 regulates the function of naïve B cells in TME. Previous studies have also shown that naïve B cells are down-regulated in advanced NSCLC and are related to poor prognosis[36]. Furthermore, CARE database analysis showed that BTNL9 expression is to be associated with effective target therapy response (Fig. 6, and Table 3).

\section{Conclusions}

In conclusion, this study shows that BTNL9 is lowly expressed in LUAD, and the low expression is associated with poor prognosis. BTNL9 may be regulated by both epigenetic regulation and post-transcriptional modification. These regulatory mechanisms may change the TME

Page 6/22 
and result in a poor prognostic phenotype and drug resistance. Therefore BTNL9 may play a pivotal role in anti-immune suppression by affecting the TME and cab be considered as a prognostic marker and new immuno-target for LUAD.

\section{Abbreviations}

LUAD: lung adenocarcinoma; LUSC:Iung squamous cell carcinoma; NSCLC:non-small cell lung cancer; BTN:Butyrophilin; BTLN:butyrophilinlike; GEPIA:gene expression profiling interactive analysis; TIMER:tumor immune estimation resource; CARE:Computational Analysis of REsistance; GEO:gene expression omnibus; ECOG:Eastern Cooperative Oncology Group; DNMTs:methyltransferases; GSEA:gene set enrichment analysis; scRNA:single-cell RNA; SNV:single nucleotide mutations; TMB:tumor mutation burden; CCLE:Cancer Cell Line Encyclopedia; GDSC:Genomics of Drug Sensitivity in Cancer; CTRP:The Cancer Therapeutics Response Portal; TILs:tumor-infiltrating immune cells; TME:tumor microenvironment.

\section{Declarations}

\section{Ethics approval and consent to participate}

Not applicable.

\section{Consent for publication}

Yes.

\section{Availability of data and materials}

All data in this study comes from public and open source databases.

\section{Author contributions}

ZC, TD contributed to the conception and design of the study. WM, JL, ZC, NH, and SZ contributed to data collection. ZC, WM, and TD analyzed and interpreted the data. ZC drafted the manuscript. All authors approved the final version of the report.

\section{Conflicts of interest}

The authors declare no potential conflict of interest.

\section{Funding}

This work was supported by The Sixth Affiliated Hospital of Guangzhou Medical University, Qingyuan People's Hospital (No project lot number).

\section{Acknowledgments}

Thanks to Hangzhou Mugu Technology Co., Ltd. for providing the open source Sangerbox tool.

\section{References}

1. Bray F, Ferlay J, Soerjomataram I, Siegel RL, Torre LA, Jemal A: Global cancer statistics 2018: GLOBOCAN estimates of incidence and mortality worldwide for 36 cancers in 185 countries. CA: a cancer journal for clinicians 2018, 68(6):394-424.

2. Chen W, Zheng R, Baade PD, Zhang S, Zeng H, Bray F, Jemal A, Yu XQ, He J: Cancer statistics in China, 2015. CA: a cancer journal for clinicians 2016, 66(2):115-132.

3. Molina JR, Yang P, Cassivi SD, Schild SE, Adjei AA: Non-small cell lung cancer: epidemiology, risk factors, treatment, and survivorship. In: Mayo Clinic Proceedings: 2008: Elsevier; 2008: 584-594.

4. Lu T, Yang X, Huang Y, Zhao M, Li M, Ma K, Yin J, Zhan C, Wang Q: Trends in the incidence, treatment, and survival of patients with lung cancer in the last four decades. Cancer management and research 2019, 11:943.

5. Abeler-Dörner L, Swamy M, Williams G, Hayday AC, Bas A: Butyrophilins: an emerging family of immune regulators. Trends in immunology 2012, 33(1):34-41. 
6. Malinowska M, Tokarz-Deptuła B, Deptuła W: Butyrophilins: an important new element of resistance. Central-European journal of immunology 2017, 42(4):399.

7. Arnett HA, Viney JL: Immune modulation by butyrophilins. Nature reviews Immunology 2014, 14(8):559-569.

8. Tang Z, Li C, Kang B, Gao G, Li C, Zhang Z: GEPIA: a web server for cancer and normal gene expression profiling and interactive analyses. Nucleic acids research 2017, 45(W1):W98-W102.

9. Clough E, Barrett T: The Gene Expression Omnibus Database. Methods in molecular biology (Clifton, NJ) 2016, 1418:93-110.

10. Li T, Fu J, Zeng Z, Cohen D, Li J, Chen Q, Li B, Liu XS: TIMER2.0 for analysis of tumor-infiltrating immune cells. Nucleic acids research 2020, 48(W1):W509-w514.

11. Győrffy B, Surowiak P, Budczies J, Lánczky A: Online survival analysis software to assess the prognostic value of biomarkers using transcriptomic data in non-small-cell lung cancer. PloS one 2013, 8(12):e82241.

12. Chandrashekar DS, Bashel B, Balasubramanya SAH, Creighton CJ, Ponce-Rodriguez I, Chakravarthi B, Varambally S: UALCAN: A Portal for Facilitating Tumor Subgroup Gene Expression and Survival Analyses. Neoplasia (New York, NY) 2017, 19(8):649-658.

13. Anaya J: OncoLnc: linking TCGA survival data to mRNAs, miRNAs, and IncRNAs. PeerJ Computer Science $2016,2: e 67$.

14. Rhodes DR, Yu J, Shanker K, Deshpande N, Varambally R, Ghosh D, Barrette T, Pandey A, Chinnaiyan AM: ONCOMINE: a cancer microarray database and integrated data-mining platform. Neoplasia (New York, NY) 2004, 6(1):1-6.

15. Kim P, Park A, Han G, Sun H, Jia P, Zhao Z: TissGDB: tissue-specific gene database in cancer. Nucleic acids research 2017, 46(D1):D1031-D1038.

16. Wickham H, Averick M, Bryan J, Chang W, Yutani H: Welcome to the Tidyverse. The Journal of Open Source Software 2019, 4(43):1686.

17. Null RCTR, Team R, Null RCT, Core Writing T, Null R, ., Team R, Null RDCT, Core R, Team R, Team RDC: R: A language and environment for statistical computing. Computing 2011, 1:12-21.

18. Tang Z, Kang B, Li C, Chen T, Zhang Z: GEPIA2: an enhanced web server for large-scale expression profiling and interactive analysis. Nucleic acids research 2019, 47(W1):W556-W560.

19. Li T, Fan J, Wang B, Traugh N, Chen Q, Liu JS, Li B, Liu XS: TIMER: A Web Server for Comprehensive Analysis of Tumor-Infiltrating Immune Cells. Cancer research 2017, 77(21):e108-e110.

20. Dai D, Chen B, Feng Y, Wang W, Jiang Y, Huang H, Liu J: Prognostic value of prostaglandin I2 synthase and its correlation with tumorinfiltrating immune cells in lung cancer, ovarian cancer, and gastric cancer. Aging 2020, 12(10):9658-9685.

21. Vejnar CE, Blum M, Zdobnov EM: miRmap web: Comprehensive microRNA target prediction online. Nucleic acids research 2013, 41(Web Server issue):W165-W168.

22. Agarwal V, Bell GW, Nam JW, Bartel DP: Predicting effective microRNA target sites in mammalian mRNAs. eLife $2015,4$.

23. Sticht C, De La Torre C, Parveen A, Gretz N: miRWalk: An online resource for prediction of microRNA binding sites. PloS one 2018, 13(10):e0206239-e0206239.

24. Li J-H, Liu S, Zhou H, Qu L-H, Yang J-H: starBase v2.0: decoding miRNA-ceRNA, miRNA-ncRNA and protein-RNA interaction networks from large-scale CLIP-Seq data. Nucleic acids research 2013, 42(D1):D92-D97.

25. Mizuno H, Kitada K, Nakai K, Sarai A: PrognoScan: a new database for meta-analysis of the prognostic value of genes. BMC Medical Genomics 2009, 2(1):18.

26. Agarwal V, Bell GW, Nam J-W, Bartel DP: Predicting effective microRNA target sites in mammalian mRNAs. eLife 2015, 4:e05005.

27. Li Y, Li L, Wang Z, Pan T, Sahni N, Jin X, Wang G, Li J, Zheng X, Zhang Y et al: LncMAP: Pan-cancer atlas of long noncoding RNAmediated transcriptional network perturbations. Nucleic acids research 2018, 46(3):1113-1123.

28. Wang P, Li X, Gao Y, Guo Q, Wang Y, Fang Y, Ma X, Zhi H, Zhou D, Shen W: LncACTdb 2.0: an updated database of experimentally supported ceRNA interactions curated from low-and high-throughput experiments. Nucleic acids research 2019, 47(D1):D121-D127.

29. Szklarczyk D, Gable AL, Lyon D, Junge A, Wyder S, Huerta-Cepas J, Simonovic M, Doncheva NT, Morris JH, Bork P et al: STRING v11: protein-protein association networks with increased coverage, supporting functional discovery in genome-wide experimental datasets. Nucleic acids research 2018, 47(D1):D607-D613.

30. Shannon P, Markiel A, Ozier O, Baliga NS, Wang JT, Ramage D, Amin N, Schwikowski B, Ideker T: Cytoscape: a software environment for integrated models of biomolecular interaction networks. Genome research 2003, 13(11):2498-2504.

31. Li Y, Xie P, Lu L, Wang J, Diao L, Liu Z, Guo F, He Y, Liu Y, Huang Q et al: An integrated bioinformatics platform for investigating the human E3 ubiquitin ligase-substrate interaction network. Nature Communications 2017, 8(1):347.

32. Faubert B, Solmonson A, DeBerardinis RJ: Metabolic reprogramming and cancer progression. Science (New York, NY) 2020, 368(6487). 
33. Yuan H, Yan M, Zhang G, Liu W, Deng C, Liao G, Xu L, Luo T, Yan H, Long Z et al: CancerSEA: a cancer single-cell state atlas. Nucleic acids research 2018, 47(D1):D900-D908.

34. Giraldo NA, Sanchez-Salas R, Peske JD, Vano Y, Becht E, Petitprez F, Validire P, Ingels A, Cathelineau X, Fridman WH et al: The clinical role of the TME in solid cancer. British Journal of Cancer 2019, 120(1):45-53.

35. Brown CC, Gudjonson H, Pritykin Y, Deep D, Lavallée V-P, Mendoza A, Fromme R, Mazutis L, Ariyan C, Leslie C et al: Transcriptional Basis of Mouse and Human Dendritic Cell Heterogeneity. Cell 2019, 179(4):846-863.e824.

36. Chen J, Tan Y, Sun F, Hou L, Zhang C, Ge T, Yu H, Wu C, Zhu Y, Duan L et al: Single-cell transcriptome and antigen-immunoglobin analysis reveals the diversity of B cells in non-small cell lung cancer. Genome Biology 2020, 21(1):152.

37. Jiang P, Lee W, Li X, Johnson C, Liu JS, Brown M, Aster JC, Liu XS: Genome-Scale Signatures of Gene Interaction from Compound Screens Predict Clinical Efficacy of Targeted Cancer Therapies. Cell systems 2018, 6(3):343-354.e345.

38. Cheng Y, He C, Wang M, Ma X, Mo F, Yang S, Han J, Wei X: Targeting epigenetic regulators for cancer therapy: mechanisms and advances in clinical trials. Signal transduction and targeted therapy 2019, 4:62.

39. Hsu Y-L, Hung J-Y, Lee Y-L, Chen F-W, Chang K-F, Chang W-A, Tsai Y-M, Chong I-W, Kuo P-L: Identification of novel gene expression signature in lung adenocarcinoma by using next-generation sequencing data and bioinformatics analysis. Oncotarget 2017 , 8(62):104831.

40. Miller DM, Thomas SD, Islam A, Muench D, Sedoris K: c-Myc and cancer metabolism. Clin Cancer Res 2012, 18(20):5546-5553.

41. Valvezan AJ, Manning BD: Molecular logic of mTORC1 signalling as a metabolic rheostat. Nature Metabolism 2019, 1(3):321-333.

42. Öjlert Å K, Halvorsen AR, Nebdal D, Lund-Iversen M, Solberg S, Brustugun OT, Lingjaerde OC, Helland $\AA$ : The immune microenvironment in non-small cell lung cancer is predictive of prognosis after surgery. Molecular oncology 2019, 13(5):1166-1179.

43. Mao Y, Feng Q, Zheng P, Yang L, Liu T, Xu Y, Zhu D, Chang W, Ji M, Ren L et al: Low tumor purity is associated with poor prognosis, heavy mutation burden, and intense immune phenotype in colon cancer. Cancer Manag Res 2018, 10:3569-3577.

44. Hu X, Zhang J, Wang J, Fu J, Li T, Zheng X, Wang B, Gu S, Jiang P, Fan J et al: Landscape of B cell immunity and related immune evasion in human cancers. Nature Genetics 2019, 51(3):560-567.

45. Böttcher JP, Reis e Sousa C: The Role of Type 1 Conventional Dendritic Cells in Cancer Immunity. Trends in Cancer 2018, 4(11):784792.

46. Perez CR, De Palma M: Engineering dendritic cell vaccines to improve cancer immunotherapy. Nature communications 2019 , 10(1):5408-5408.

\section{Tables}

Table 1. BTNL9 mRNA expression and characteristics in TCGA-LUAD 


\begin{tabular}{|c|c|c|c|}
\hline \multirow[t]{2}{*}{ Characteristics } & \multicolumn{3}{|l|}{ BTNL9 mRNA } \\
\hline & High (N=291) & Low (N=294) & Overall \\
\hline \multicolumn{4}{|l|}{ Age (years) } \\
\hline$<40$ & $1(0.3 \%)$ & $3(1.0 \%)$ & $4(0.7 \%)$ \\
\hline $40-60$ & 77 (26.4\%) & $101(34.5 \%)$ & $178(30.4 \%)$ \\
\hline$>60$ & 208 (71.2\%) & $176(60.1 \%)$ & $384(65.6 \%)$ \\
\hline \multicolumn{4}{|l|}{ Sex } \\
\hline Female & 165 (56.5\%) & $151(51.5 \%)$ & $316(54.0 \%)$ \\
\hline Male & 127 (43.5\%) & $142(48.5 \%)$ & 269 (46.0\%) \\
\hline \multicolumn{4}{|l|}{ Race } \\
\hline Asian & $5(1.7 \%)$ & $2(0.7 \%)$ & $7(1.2 \%)$ \\
\hline Black & $30(10.3 \%)$ & $28(9.6 \%)$ & $58(9.9 \%)$ \\
\hline White & 232 (79.5\%) & $221(75.4 \%)$ & $453(77.4 \%)$ \\
\hline \multicolumn{4}{|l|}{ T stage } \\
\hline T1 & 115 (39.4\%) & 76 (25.9\%) & 191 (32.6\%) \\
\hline T2 & 145 (49.7\%) & $176(60.1 \%)$ & $321(54.9 \%)$ \\
\hline T3 & $20(6.8 \%)$ & $30(10.2 \%)$ & $50(8.5 \%)$ \\
\hline T4 & $10(3.4 \%)$ & $10(3.4 \%)$ & $20(3.4 \%)$ \\
\hline \multicolumn{4}{|l|}{$\mathrm{N}$ stage } \\
\hline NO & 199 (68.2\%) & $172(58.7 \%)$ & $371(63.4 \%)$ \\
\hline $\mathrm{N} 1$ & $46(15.8 \%)$ & $61(20.8 \%)$ & 107 (18.3\%) \\
\hline N2-N3 & $35(12.0 \%)$ & $54(18.4 \%)$ & $89(15.2 \%)$ \\
\hline \multicolumn{4}{|l|}{ M stage } \\
\hline M0 & 192 (65.8\%) & 202 (68.9\%) & $394(67.4 \%)$ \\
\hline M1 & $12(4.1 \%)$ & $15(5.1 \%)$ & $27(4.6 \%)$ \\
\hline \multicolumn{4}{|l|}{ ECOG } \\
\hline $0-1$ & $60(20.5 \%)$ & $54(18.4 \%)$ & 114 (19.5\%) \\
\hline $2-3$ & $15(5.1 \%)$ & $10(3.4 \%)$ & $25(4.3 \%)$ \\
\hline 4 & $1(0.3 \%)$ & $1(0.3 \%)$ & $2(0.3 \%)$ \\
\hline \multicolumn{4}{|l|}{ EGFR mutation } \\
\hline No & $113(38.7 \%)$ & 99 (33.8\%) & \\
\hline Yes & 47 (16.1\%) & 39 (13.3\%) & \\
\hline \multicolumn{4}{|l|}{ KRAS mutation } \\
\hline No & $21(7.2 \%)$ & $23(7.8 \%)$ & $44(7.5 \%)$ \\
\hline Yes & $17(5.8 \%)$ & $9(3.1 \%)$ & $26(4.4 \%)$ \\
\hline \multicolumn{4}{|c|}{ Radiation therapy } \\
\hline No & 45 (15.4\%) & 46 (15.7\%) & 91 (15.6\%) \\
\hline Yes & 32 (11.0\%) & 38 (13.0\%) & $70(12.0 \%)$ \\
\hline
\end{tabular}

Page 10/22 
Table 2. Correlation analysis between BTNL9 and relate gene set markers of significant innate and adaptive immunity cells in TIMER and GEPIA database 


\begin{tabular}{|c|c|c|c|c|c|c|c|c|c|}
\hline \multirow{3}{*}{$\begin{array}{l}\text { Immune } \\
\text { cell }\end{array}$} & \multirow{3}{*}{ Marker } & \multicolumn{4}{|l|}{ TIMER } & \multicolumn{4}{|l|}{ GEPIA } \\
\hline & & \multicolumn{2}{|l|}{ None } & \multicolumn{2}{|l|}{ purity } & \multicolumn{2}{|l|}{ Normal } & \multicolumn{2}{|l|}{ Cancer } \\
\hline & & $\begin{array}{l}\text { Spearman's } \\
\rho\end{array}$ & P Value & $\begin{array}{l}\text { Spearman's } \\
\rho\end{array}$ & $\begin{array}{l}\mathrm{P} \\
\text { Value }\end{array}$ & $\begin{array}{l}\text { Spearman } \\
\text { correlation } \\
\text { coefficient }\end{array}$ & $\begin{array}{l}\mathrm{P} \\
\text { Value }\end{array}$ & $\begin{array}{l}\text { Spearman } \\
\text { correlation } \\
\text { coefficient }\end{array}$ & $\begin{array}{l}\mathrm{P} \\
\text { Value }\end{array}$ \\
\hline \multirow[t]{8}{*}{ DC } & CD1C & 0.36 & $1.65 \mathrm{E}-17$ & 0.34 & $\begin{array}{l}1.03 \mathrm{E}- \\
14\end{array}$ & -0.022 & 0.87 & 0.33 & $\begin{array}{l}1.5 \mathrm{E}- \\
13^{-}\end{array}$ \\
\hline & HLA-DPA1 & 0.23 & $1.64 \mathrm{E}-07$ & 0.20 & $\begin{array}{l}8.47 \mathrm{E}- \\
06\end{array}$ & & & & \\
\hline & HLA-DPB1 & 0.29 & 8.39E-12 & 0.27 & $\begin{array}{l}8.03 \mathrm{E}- \\
10\end{array}$ & & & & \\
\hline & HLA-DQB1 & 0.21 & $1.31 \mathrm{E}-06$ & 0.18 & $\begin{array}{l}8.16 \mathrm{E}- \\
05\end{array}$ & & & & \\
\hline & HLA-DRA & 0.18 & $4.54 \mathrm{E}-05$ & 0.14 & 0.0020 & & & & \\
\hline & ITGAX & 0.24 & 1.63E-08 & 0.22 & $\begin{array}{l}1.10 \mathrm{E}- \\
06\end{array}$ & & & & \\
\hline & NCR1 & 0.04 & 0.35980601 & 0.01 & 0.8450 & & & & \\
\hline & NRP1 & 0.03 & 0.41981037 & 0.03 & 0.4973 & & & & \\
\hline \multirow[t]{3}{*}{ cDC1s } & CD8A & 0.03 & 0.55316497 & -0.03 & 0.5613 & -0.028 & 0.83 & 0.22 & $\begin{array}{l}1.20 \mathrm{E}- \\
06\end{array}$ \\
\hline & CLEC9A & 0.40 & $1.00 \mathrm{E}-21$ & 0.38 & $\begin{array}{l}1.16 \mathrm{E}- \\
18\end{array}$ & & & & \\
\hline & XCR1 & 0.41 & $2.70 \mathrm{E}-22$ & 0.39 & $\begin{array}{l}7.37 \mathrm{E}- \\
20\end{array}$ & & & & \\
\hline \multirow[t]{2}{*}{ cDC2s } & CLEC12A & 0.22 & $3.50 \mathrm{E}-07$ & 0.18 & $\begin{array}{l}3.61 \mathrm{E}- \\
05\end{array}$ & 0.18 & 0.16 & 0.45 & $\begin{array}{l}7.1 \mathrm{E}- \\
25\end{array}$ \\
\hline & ESAM & 0.51 & $1.01 \mathrm{E}-35$ & 0.50 & $\begin{array}{l}2.89 \mathrm{E}- \\
33\end{array}$ & & & & \\
\hline \multirow[t]{4}{*}{ B cell } & CD19 & 0.23 & 1.93E-07 & 0.20 & $\begin{array}{l}5.30 \mathrm{E}- \\
06\end{array}$ & 0.017 & $\begin{array}{l}9.00 \mathrm{E}- \\
01\end{array}$ & 0.37 & $\begin{array}{l}8.30 \mathrm{E}- \\
17\end{array}$ \\
\hline & FCER2 & 0.40 & 7.97E-21 & 0.38 & $\begin{array}{l}2.18 \mathrm{E}- \\
18\end{array}$ & & & & \\
\hline & MS4A1 & 0.34 & $6.45 \mathrm{E}-16$ & 0.33 & $\begin{array}{l}2.59 \mathrm{E}- \\
14\end{array}$ & & & & \\
\hline & SDC1 & 0.07 & 0.0917 & 0.09 & 0.0523 & & & & \\
\hline \multirow[t]{5}{*}{$\begin{array}{l}\text { Naïve B } \\
\text { cell }\end{array}$} & CD19 & 0.23 & $1.93 \mathrm{E}-07$ & 0.20 & $\begin{array}{l}5.30 \mathrm{E}- \\
06\end{array}$ & 0.082 & 0.54 & 0.43 & $\begin{array}{l}8.60 \mathrm{E}- \\
23\end{array}$ \\
\hline & CD22 & 0.50 & $2.44 \mathrm{E}-34$ & 0.51 & $\begin{array}{l}1.10 \mathrm{E}- \\
34\end{array}$ & & & & \\
\hline & CD83 & 0.33 & $6.56 \mathrm{E}-15$ & 0.31 & $\begin{array}{l}2.09 \mathrm{E}- \\
12\end{array}$ & & & & \\
\hline & MS4A1 & 0.34 & $6.45 \mathrm{E}-16$ & 0.33 & $\begin{array}{l}2.59 \mathrm{E}- \\
14\end{array}$ & & & & \\
\hline & TCL1A & 0.25 & $1.57 \mathrm{E}-08$ & 0.21 & $\begin{array}{l}1.31 \mathrm{E}- \\
06\end{array}$ & & & & \\
\hline \multirow[t]{2}{*}{$\begin{array}{l}\text { Plasma } \\
\text { B cell }\end{array}$} & CD38 & 0.04 & 0.3699 & 0.08 & 0.0746 & -0.19 & 0.15 & 0.059 & 0.19 \\
\hline & TNFRSF17 & 0.04 & 0.4053 & 0.08 & 0.0814 & & & & \\
\hline
\end{tabular}


Table 3. Loss of BTNL9 expression may promote drug resistance toward many targeted therapies in CGP, CCLE, and CTRP cohorts 


\begin{tabular}{|c|c|c|c|c|}
\hline & Drug & Target & t-value & $\mathrm{p}$-value \\
\hline & $10 \times 2$ & EGLN1 & 5.91996 & 4.56E-09 \\
\hline & PHA-793887 & CDK9 & 4.92172 & $1.02 \mathrm{E}-06$ \\
\hline & OSI-027 & MTOR & 4.55428 & 5.99E-06 \\
\hline & Ispinesib & KIF11 & 4.42265 & 1.09E-05 \\
\hline & Nilotinib & ABL1 & 4.3886 & 1.30E-05 \\
\hline & Axitinib & PDGFRA & 4.33456 & 1.64E-05 \\
\hline & NG25 & MAP4K2 & 4.29746 & $1.92 \mathrm{E}-05$ \\
\hline & Nilotinib & KIT & 4.26379 & $2.26 \mathrm{E}-05$ \\
\hline & BMS345541 & IKBKB & 4.12048 & 4.13E-05 \\
\hline & GSK525762A & BRD2 & 3.92075 & $9.50 \mathrm{E}-05$ \\
\hline & CAY10603 & HDAC6 & 3.91591 & 9.69E-05 \\
\hline & TubastatinA & HDAC6 & 3.89552 & 0.000105 \\
\hline & GSK525762A & BRD4 & 3.83187 & 0.000136 \\
\hline CGP dataset & PHA-793887 & CDK1 & 3.4184 & 0.000658 \\
\hline & Fluorouracil & TYMS & 3.09815 & 0.002008 \\
\hline & TPCA-1 & IKBKB & 3.09042 & 0.002061 \\
\hline & $1256580-46-7$ & ALK & 3.01459 & 0.002646 \\
\hline & CAL-101 & PIK3CD & 2.99145 & 0.002852 \\
\hline & Belinostat & HDAC6 & 2.82403 & 0.004852 \\
\hline & AT7519 & CDK9 & 2.81016 & 0.00506 \\
\hline & CP-466722 & ATM & 2.78047 & 0.005542 \\
\hline & Enzastaurin & PRKCB & 2.76951 & 0.00573 \\
\hline & SB590885 & BRAF_V600E.Mutation & 2.6446 & 0.008337 \\
\hline & Vorinostat & HDAC6 & 2.54112 & 0.011233 \\
\hline & Nutlin-3 & MDM2 & -2.94731 & 0.003295 \\
\hline & Dasatinib & EPHA2 & -3.23912 & 0.001305 \\
\hline & Quizartinib & FLT3 & -3.33865 & 0.000877 \\
\hline & 1173900-33-8 & PIK3CB & -3.55986 & 0.00039 \\
\hline & Linifanib & FLT3 & -5.1822 & $2.71 \mathrm{E}-07$ \\
\hline & $870483-87-7$ & CSF1R & -9.60837 & 7.14E-21 \\
\hline & Panobinostat & HDAC1 & 3.29253 & 0.001066 \\
\hline & abraxane & TUBB & 2.83794 & 0.00473 \\
\hline CCLE dataset & Palbociclib & RB1 & 2.64989 & 0.008358 \\
\hline & Topotecan & TOP1 & 2.45344 & 0.014498 \\
\hline & Sorafenib & FLT3 & -4.25026 & $2.56 \mathrm{E}-05$ \\
\hline & 9-Fluoro-11,17,21-trihydroxy-16-methylpregna-1,4-diene-3,20-dione & NR3C1 & 5.30047 & $1.51 \mathrm{E}-07$ \\
\hline & abraxane & TUBB & 4.50938 & 7.51E-06 \\
\hline & Alisertib & AURKB & 4.50069 & 7.83E-06 \\
\hline
\end{tabular}

Page $14 / 22$ 


\begin{tabular}{|c|c|c|c|c|}
\hline & PAC-1 & CASP3 & 4.27611 & 2.16E-05 \\
\hline & $660868-91-7$ & PLK1 & 4.21764 & 2.79E-05 \\
\hline & Gossypol & BCL2 & 4.19093 & 3.12E-05 \\
\hline & Decitabine & DNMT1 & 4.18042 & $3.24 \mathrm{E}-05$ \\
\hline & 722544-51-6 & AURKB & 4.14645 & $3.74 \mathrm{E}-05$ \\
\hline & Etoposide & TOP2B & 4.0066 & $6.76 \mathrm{E}-05$ \\
\hline & 3,5-bis(4-methylbenzylidene)piperidin-4-one & USP13 & 3.92444 & $9.47 \mathrm{E}-05$ \\
\hline & $180002-83-9$ & CNR2 & 3.91953 & $9.68 \mathrm{E}-05$ \\
\hline & CICLOPIROX & RRM1 & 3.90668 & 0.000102 \\
\hline & Dabrafenib & BRAF_V600E.Mutation & 3.84863 & 0.00014 \\
\hline & BRD-K62801835-001-01-0 & $\mathrm{EZH} 2$ & 3.80929 & 0.000151 \\
\hline & BI2536 & PLK1 & 3.69725 & 0.000233 \\
\hline & Nilotinib & ABL1 & 3.67464 & 0.000255 \\
\hline & Cerulenin & HMGCS1 & 3.66163 & 0.000268 \\
\hline & TW-37 & BCL2 & 3.585 & 0.000358 \\
\hline & zebularine & DNMT1 & 3.57912 & 0.000366 \\
\hline & Pevonedistat & NAE1 & 3.55057 & 0.000409 \\
\hline & BAS02002358 & GPER1 & 3.52883 & 0.000442 \\
\hline & KU-60019 & ATM & 3.52842 & 0.000443 \\
\hline & narciclasine & $\mathrm{RHOA}$ & 3.47702 & 0.000536 \\
\hline & Ki8751 & PDGFRA & 3.43765 & 0.000619 \\
\hline & 4ly1 & HDAC1 & 3.39584 & 0.000719 \\
\hline CTRP dataset & SCHEMBL12182311 & EIF4E & 3.35831 & 0.000822 \\
\hline & Belinostat & HDAC1 & 3.35122 & 0.000886 \\
\hline & Masitinib & PDGFRA & 3.32101 & 0.000941 \\
\hline & BIBR1532 & TERT & 3.31812 & 0.000949 \\
\hline & UNII-UZ77T1VFBM & BIRC5 & 3.30878 & 0.000985 \\
\hline & Nutlin-3 & MDM2 & 3.2982 & 0.001018 \\
\hline & CHEMBL2058177 & EIF4E & 3.29052 & 0.001045 \\
\hline & NSC373989 & MDM2 & 3.24218 & 0.001239 \\
\hline & Imatinib & ABL1 & 3.22213 & 0.001327 \\
\hline & Tacrolimus & РРP3СВ & 3.13945 & 0.001761 \\
\hline & Olaparib & PARP1 & 3.07789 & 0.002159 \\
\hline & SMR001317659 & PDE4A & 3.03729 & 0.002467 \\
\hline & Axitinib & PDGFRA & 3.02572 & 0.002563 \\
\hline & Pubchem_92131101 & KIF11 & 3.01909 & 0.002617 \\
\hline & abraxane & TUBB1 & 3.01218 & 0.002678 \\
\hline & GSK461364 & PLK1 & 2.97879 & 0.002987 \\
\hline
\end{tabular}

Page 15/22 


\begin{tabular}{|c|c|c|c|}
\hline Sorafenib & PDGFRA & 2.93438 & 0.003443 \\
\hline Nutlin-3 & TP53 & 2.88952 & 0.003968 \\
\hline SMR000068650 & S1PR2 & 2.88871 & 0.003992 \\
\hline MK-1775 & WEE1 & 2.88827 & 0.003985 \\
\hline BRD-K53855319-001-01-2 & SIRT1 & 2.87063 & 0.004217 \\
\hline Apicidin & HDAC1 & 2.7849 & 0.005485 \\
\hline TelomeraselnhibitorlX & TERT & 2.7825 & 0.005527 \\
\hline Pluripotin & MAPK1 & 2.76988 & 0.005741 \\
\hline PHA-793887 & CDK1 & 2.75543 & 0.005999 \\
\hline Vorinostat & HDAC1 & 2.74595 & 0.006174 \\
\hline I-BET151 & BRD4 & 2.73756 & 0.006332 \\
\hline SCHEMBL12182311 & EIF4A2 & 2.68306 & 0.007448 \\
\hline $112522-64-2$ & HDAC2 & 2.66828 & 0.007855 \\
\hline RG108 & DNMT1 & 2.65008 & 0.008212 \\
\hline PF184 & IKBKB & 2.63052 & 0.008701 \\
\hline Pazopanib & PDGFRA & 2.63026 & 0.008704 \\
\hline JQ-1 & BRD4 & 2.59578 & 0.009615 \\
\hline SCHEMBL13833318 & HDAC1 & 2.56094 & 0.010633 \\
\hline CAY10603 & HDAC6 & 2.55261 & 0.010887 \\
\hline GSK525762A & BRD4 & 2.51599 & 0.012071 \\
\hline 4CA-0620 & PLK1 & 2.43094 & 0.01529 \\
\hline ВСР9000801 & MDM2 & 2.41963 & 0.015783 \\
\hline Belinostat & HDAC6 & 2.37335 & 0.018128 \\
\hline Tipifarnib & FNTA & 2.36404 & 0.018536 \\
\hline prima-1 & TP53 & 2.35318 & 0.018879 \\
\hline BMS345541 & $\mathrm{IKBKB}$ & 2.35317 & 0.018872 \\
\hline Gemcitabine & RRM1 & 2.3315 & 0.019984 \\
\hline SMR001317659 & PDE4B & 2.28061 & 0.022841 \\
\hline Doxorubicin & TOP2B & 2.26369 & 0.023866 \\
\hline Rigosertib & PIK3CA & 2.22431 & 0.026433 \\
\hline 3,5-di-tert-butylchalcone & RARA & -2.40858 & 0.016249 \\
\hline Dasatinib & EPHA2 & -2.95126 & 0.003261 \\
\hline
\end{tabular}

\section{Figures}




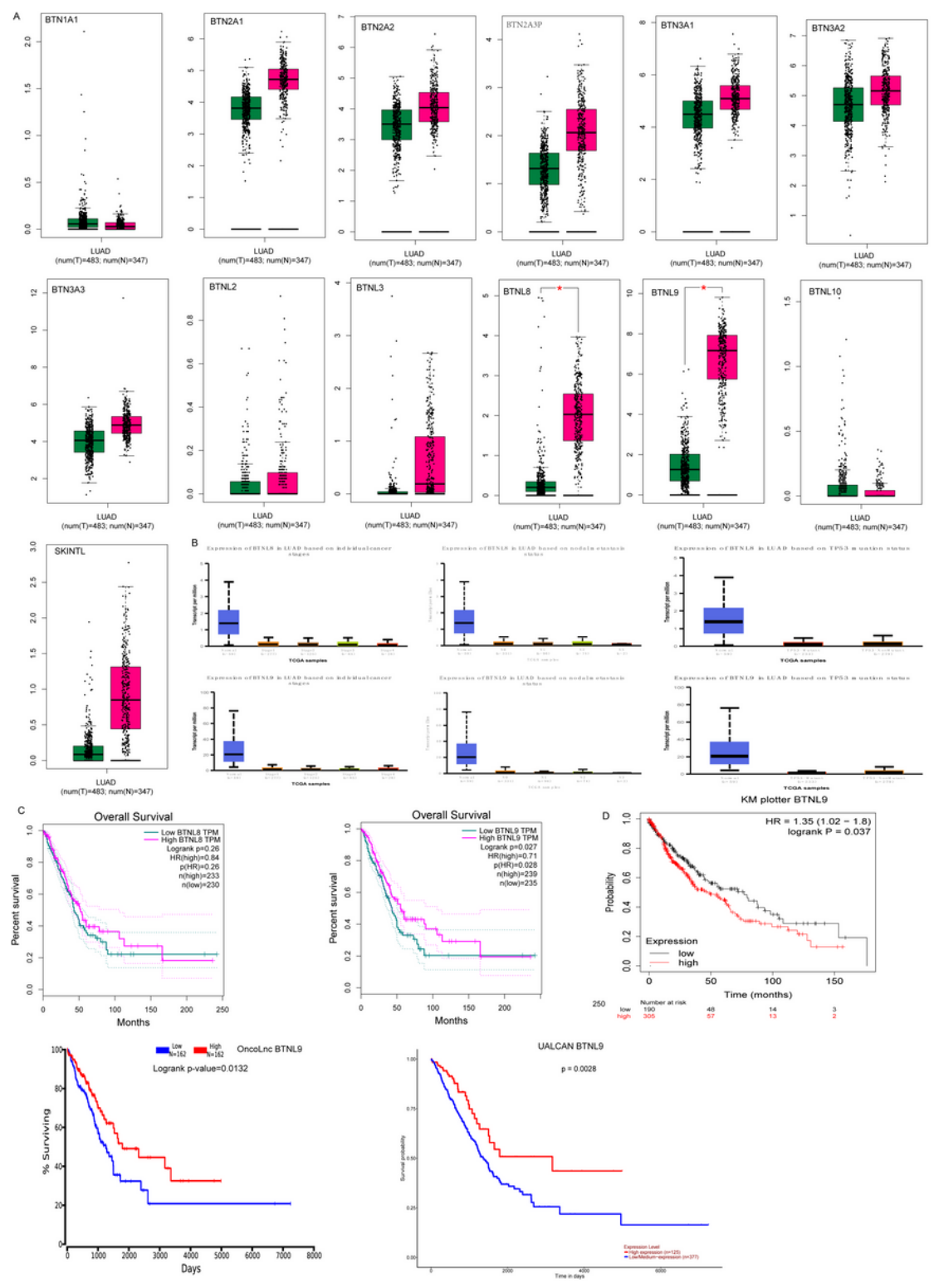

Figure 1

BTNL9 mRNA Expression and prognosis in LUAD. (A) BTN and BTNL family expression panel in LUAD compared to adjacent tissues in the GEPIA database. (B) Correlation between BTNL8 and BTNL9 expression and LUAD clinical stages, N stage, and P53 mutation in the UALCAN database (BTNL8 comparison in clinical stage (Normal-vs-Stage1, P=1.01E-04; Normal-vs-Stage2, P=7.08E-03; Normal-vs-Stage3, $\mathrm{P}=1.36 \mathrm{E}-04$; Normal-vs-Stage4, $\mathrm{P}=1.78 \mathrm{E}-08$ ), N stage (Normal-vs-N0, $\mathrm{P}=6.56 \mathrm{E}-04$; Normal-vs-N1, $\mathrm{P}=8.27 \mathrm{E}-10$; Normal-vs-N2, $\mathrm{P}=5.96 \mathrm{E}-04$; Normal-vs-N3, $P=1.97 E-10 ;$ NO-vs-N1, $P=3.27 E-03 ;$ NO-vs-N3, $P=6.79 E-04)$, and P53 status (Normal-vs-TP53-Mutant, $P=1.27 E-06$; Normal-vsTP53-NonMutant, $P=1.80 \mathrm{E}-03$ ). BTNL9 comparison in clinical stage (Normal-vs-Stage1, $P=1.21 \mathrm{E}-12$; Normal-vs-Stage2, $P=3.60 \mathrm{E}-13$; Normal-vs-Stage3, $P=2.40 E-12 ;$ Normal-vs-Stage4, $P=2.25 E-12$ ), N stage (Normal-vs-N0, $P=1.08 E-12$; Normal-vs-N1, $P=1.85 E-12 ;$ Normal-vsN2, P=1.02E-12; Normal-vs-N3, P=1.67E-12; N0-vs-N1, P=1.02E-03; N2-vs-N3, P=3.01E-02), and P53 status (Normal-vs-TP53-Mutant, $\mathrm{P}=1.85 \mathrm{E}-12$; Normal-vs-TP53-NonMutant, $\mathrm{P}=4.10 \mathrm{E}-12$; TP53-Mutant-vs-TP53-NonMutant, $\mathrm{P}=1$.77E-04)). (C) The correlation between BTNL8 and BTNL9 expression with overall survival of LUAD in GEPIA. (D) Correlation between BTNL9 expression and LUAD overall survival in KM plotter, OncoLnc, and UALCAN databases.*: P-value < 0.05; $*$ : P-value $<0.01 ; * \star *$ : P-value $<0.001$. 


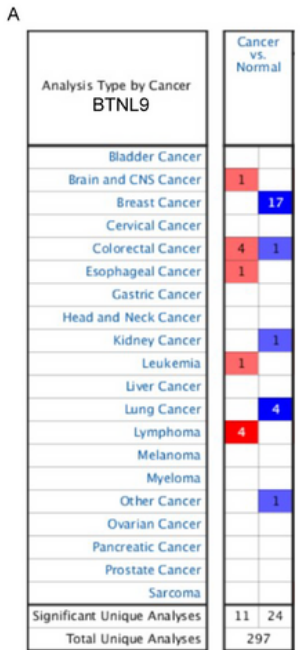

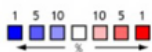

C

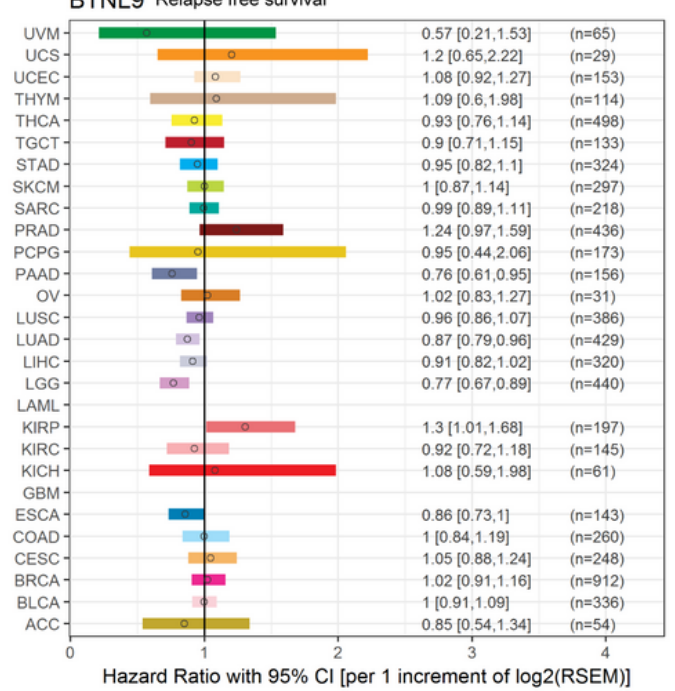

D

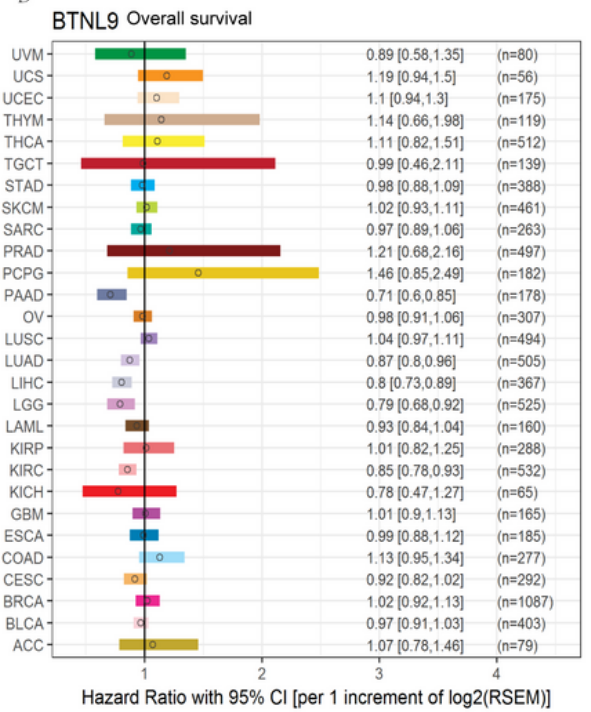

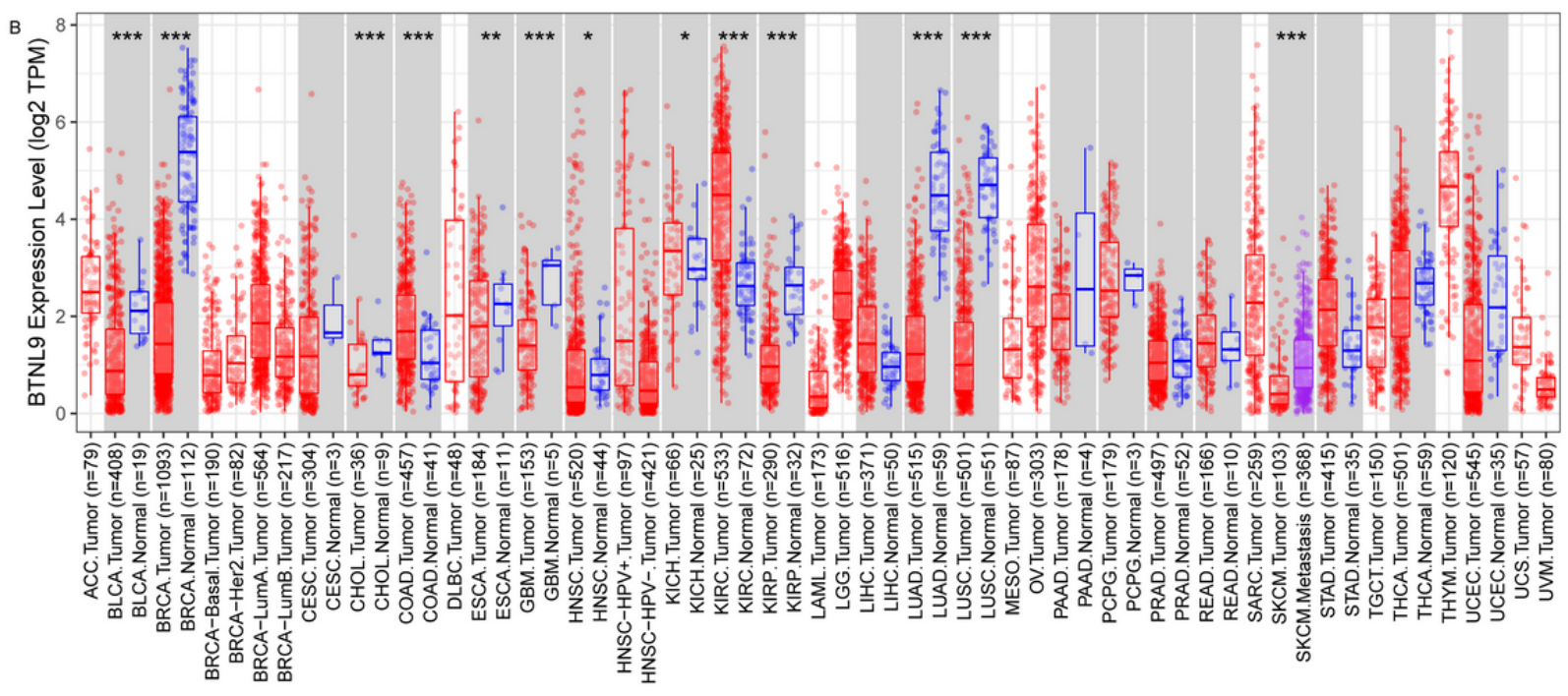

Figure 2

mRNA Expression of BTNL9 in pan-cancer and hazard ratio of relapse-free survival and overall survival,in LUAD. (A) Increased or decreased expression of BTNL9 in various tumors compared to adjacent tissues in the Oncomine database. (B) BTNL9 expression in pan-cancer inthe TCGA database analyzed by TIMER $\left({ }^{*} P<0.05,{ }^{\star *} \mathrm{P}<0.01,{ }^{\star} * \mathrm{P}<0.001\right)$. (C) BTNL9 expression and hazard ratio of relapse-free survival, and (D) overall survival. 

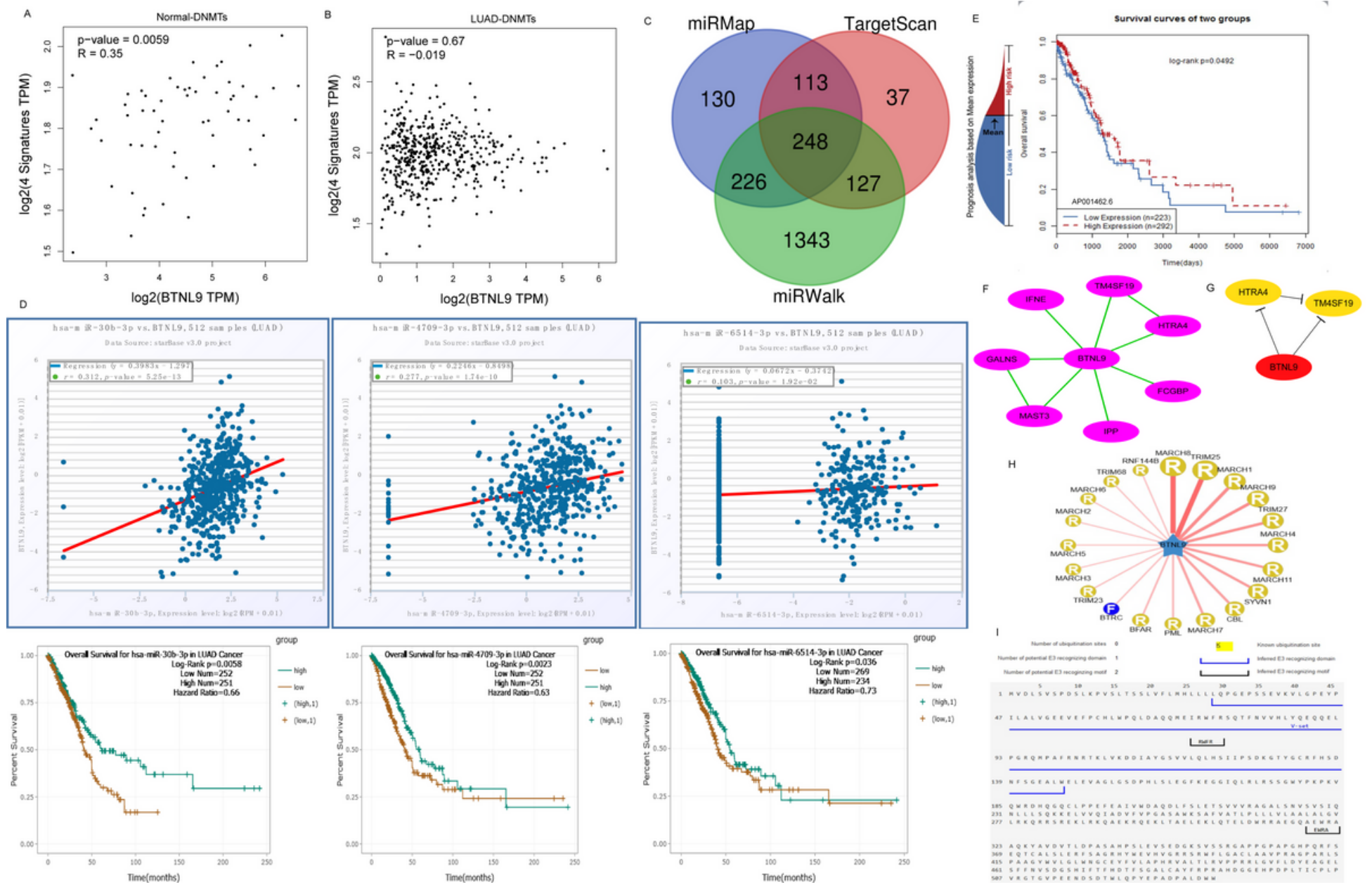

Figure 3

Epigenetic regulation and post-translation modulation network of BTNL9 in LUAD. (A, B) The correlation between BTNL9 expression and methyltransferases (DNMTs) including DNMT1, DNMT2, DNMT3A, and DNMT3B in LUAD and adjacent tissue in GEPIA. (C) Predicted miRNAs that bind to BTNL9 in miRMap, TargetScan, and miRWalk databases are shown by the Venn diagram.(D) The overlapping 248 miRNAs verified in the StarBase database, and hsa-miR-30b-3p, hsa-miR-4709-3p and hsa-miR-6514-39 were screened..(E) Predicted LncRNAs that binds to BTNL9 in the LncMap database (Supplementary Table 3). AP001462.6 was verified and screened in LncACTdb2.0 database. $(F, G)$ BTNL9 interacting protein identified usingthe STRING databaseresults edited usingCytoscape software (V3.7.2), and the hub genes screened using the cytoHubba module of Cytoscape. $(\mathrm{H}, \mathrm{I})$ Ubibrowser database predicts that the substrate BTNL 9 can be bound by E3 (MARCH8) ligases, with one potential E3 recognizing domain and two potential E3 recognizing motifs. 

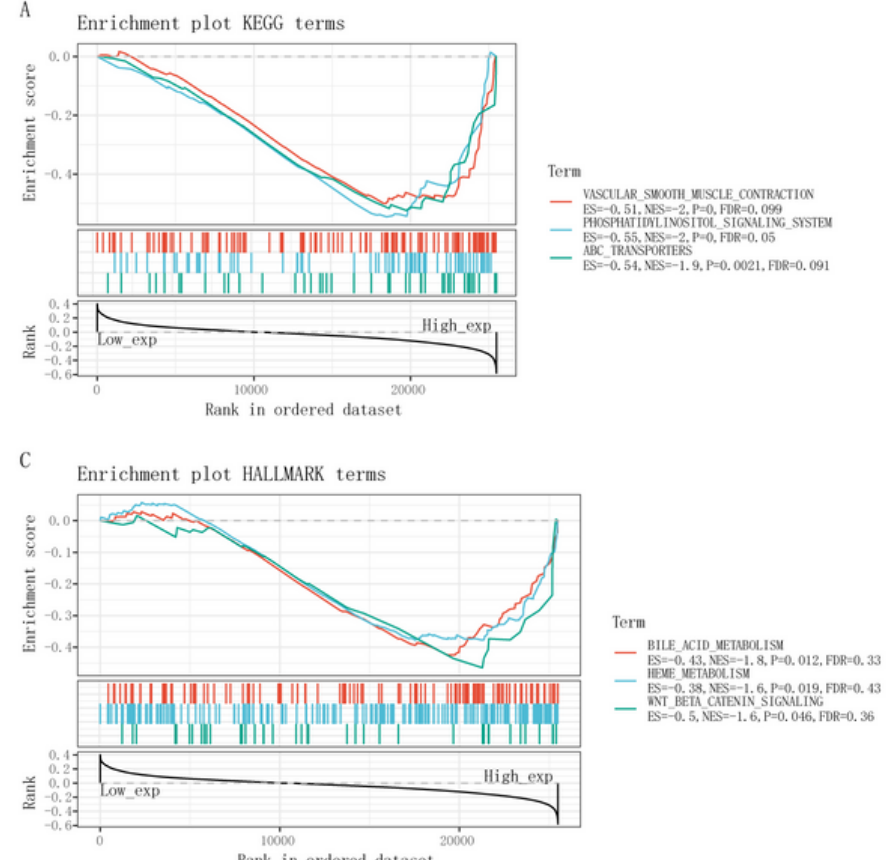

E BTNL9 Expression distribution with t-SNE plot

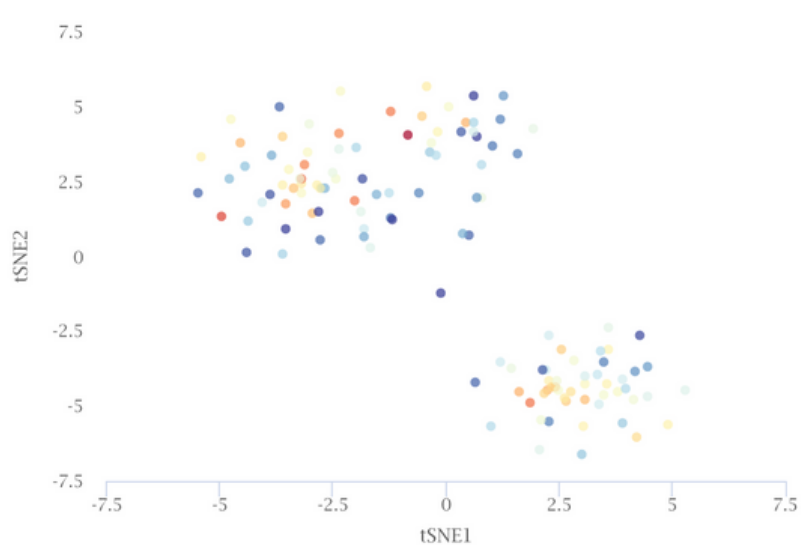

B Enrichment plot KEGG terms

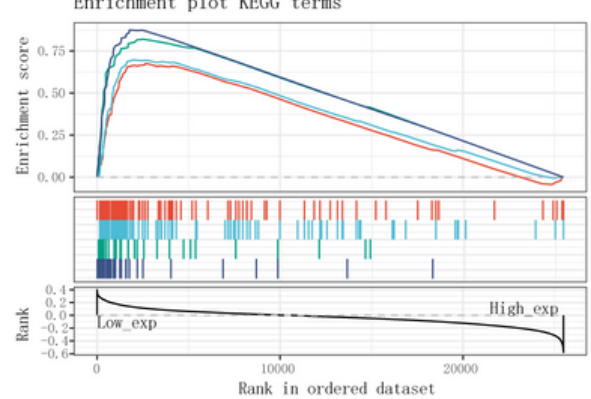

Term

PARKISSONS DISEASE

EST. $\mathrm{ES}=0.7, \mathrm{NES}=2 . \mathrm{P}=0.0019, \mathrm{FPB}=0.018$ DN. Repticanion - PROTELSOME

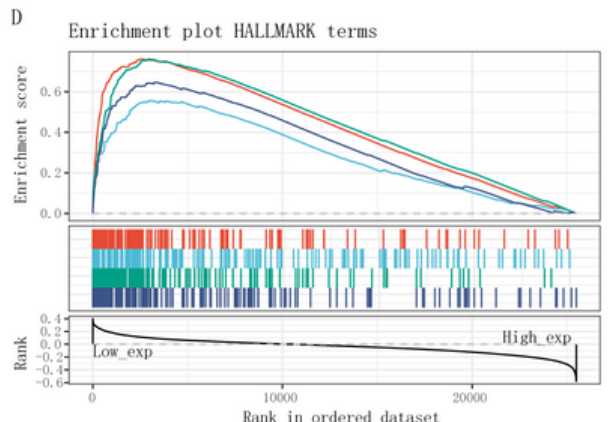

Term

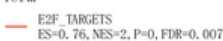

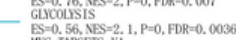

-

-
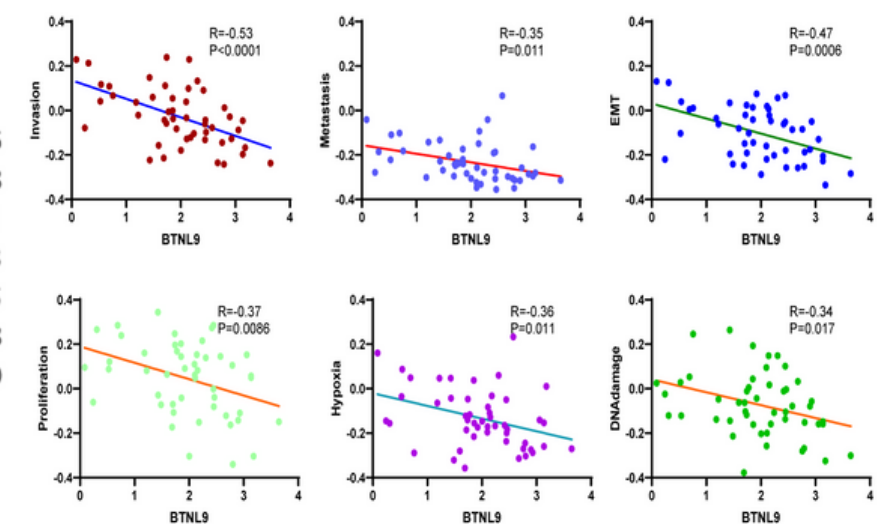

Figure 4

Low expression of BTNL9 significantly enriches proteasome and promotes tumor malignancy in LUAD. (A, B, C, D) High and low BTNL9 expression groups presented usingthe Sangerbox tool and GSEA for KEGG and HALLMARK pathways. (E) single-cell RNA (scRNA) analysis of the BTNL9 expression is shown by the t-SNE plot, and (F) molecular function of BTNL9 in LUAD using the CancerSEA database demonstrates that BTNL9 is significantly negatively correlated with tumor invasion, metastasis, EMT, proliferation, hypoxia, and DNA damage in LUAD. 
A

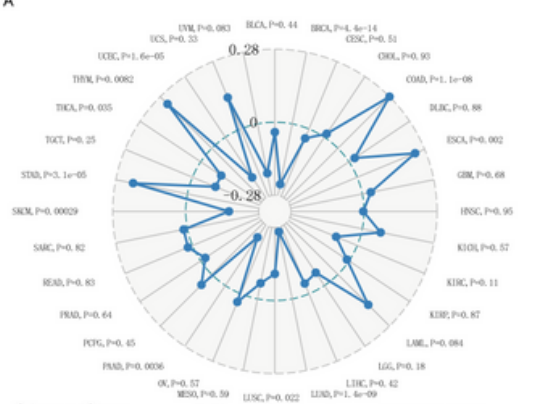

B 1$]$

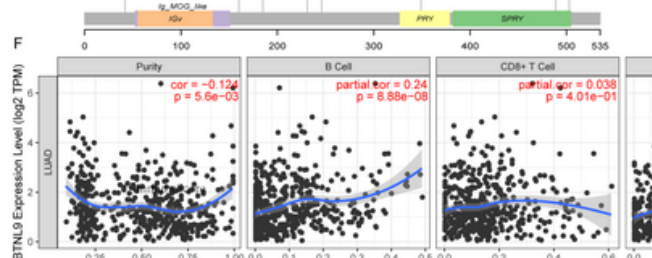

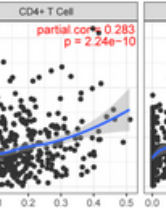
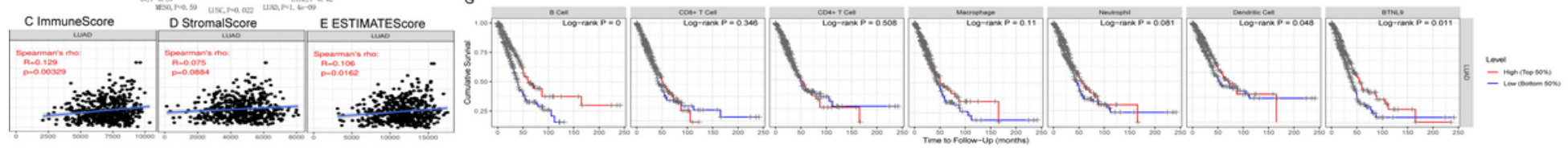

Figure 5

Correlation between BTNL9 and tumor-infiltrating immune cells and its OS prognosis. (A) Sangerbox tools analysis ofthe correlation between BTNL9 and TMB, and (B) the somatic mutation pattern of BTNL9 in LUAD, and (C, D, E) ESTIMATE scores. (F) Correlation between BTNL9 and TME infiltrating immune cells analyzed using TIMER database, and (G) overall survival.

BTNL9 expression

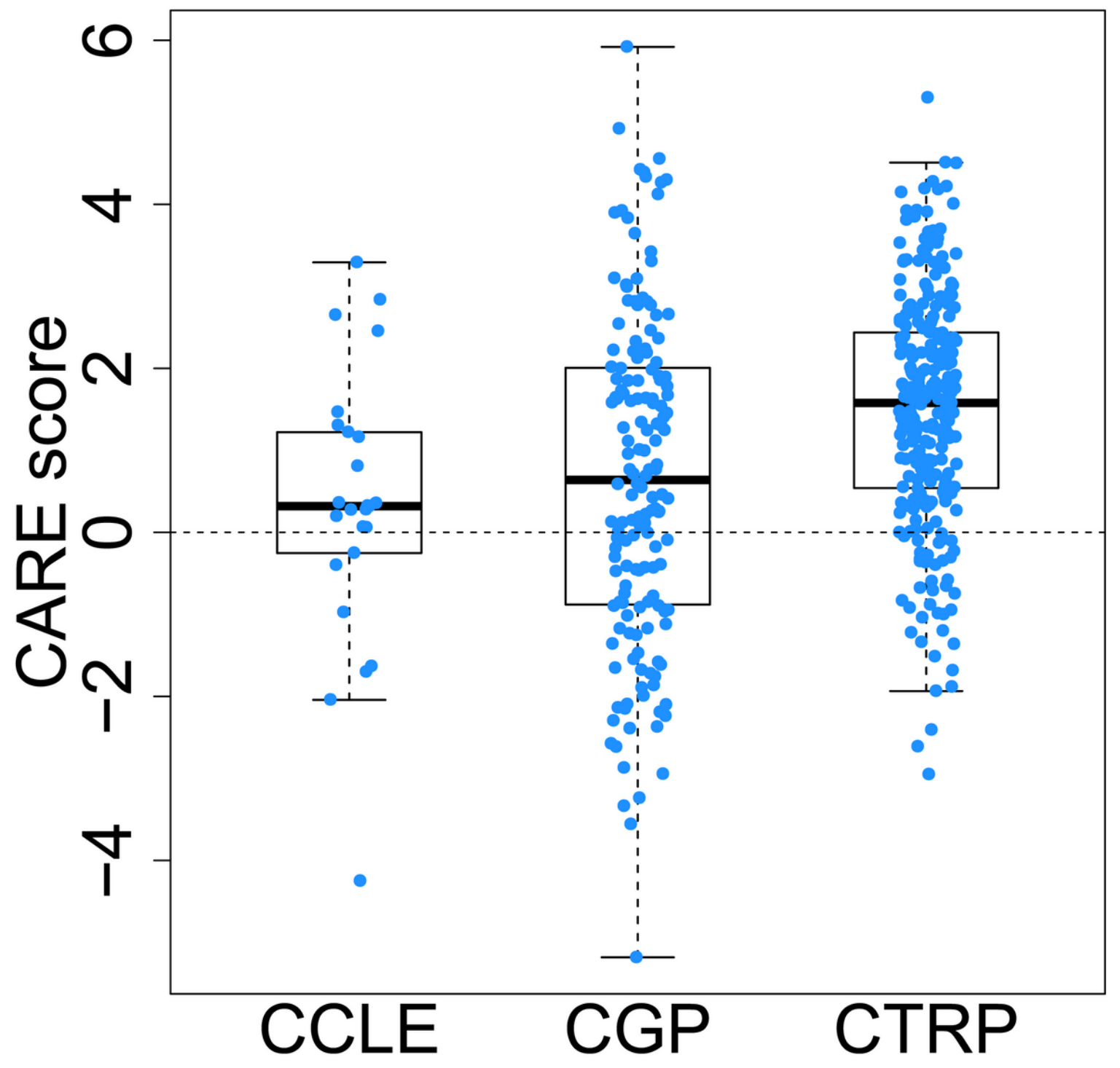




\section{Figure 6}

BTNL9 expression is associated with target therapy response BTNL9 expression has significant positive CARE scores for many compounds in Cancer Cell Line Encyclopedia (CCLE), Genomics of Drug Sensitivity in Cancer (GDSC, previously named CGP), and The Cancer Therapeutics Response Portal (CTRP) cohorts.

\section{Supplementary Files}

This is a list of supplementary files associated with this preprint. Click to download.

- SupplementaryFigure1.Flowdiagram.tif

- SupplementaryTable1.docx

- SupplementaryTable2.docx

- SupplementaryTable3.docx

- SupplementaryTable4.docx 